\title{
Effects of Resistance Training on Smoking Abstinence Self- Efficacy in Sedentary Smokers
}

\author{
Scott Richardson \\ Franklin Pierce University, richardsons@franklinpierce.edu \\ Brent Alvar \\ Point Loma Nazarene University, balvar@pointloma.edu \\ Daniel Dodd \\ Illinois State University, ddodd@ilstu.edu \\ Uma Nair \\ University of Arizona, umanair@email.arizona.edu \\ Ryan Seltzer \\ Translational Analytics and Statistics, rseltzer@trans-stat.com
}

Follow this and additional works at: https://nsuworks.nova.edu/ijahsp

Part of the Medicine and Health Sciences Commons

\section{Recommended Citation}

Richardson S, Alvar B, Dodd D, Nair U, Seltzer R. Effects of Resistance Training on Smoking Abstinence Self-Efficacy in Sedentary Smokers. The Internet Journal of Allied Health Sciences and Practice. 2020 Jan 01;18(3), Article 10.

This Manuscript is brought to you for free and open access by the College of Health Care Sciences at NSUWorks. It has been accepted for inclusion in Internet Journal of Allied Health Sciences and Practice by an authorized editor of NSUWorks. For more information, please contact nsuworks@nova.edu. 


\title{
Effects of Resistance Training on Smoking Abstinence Self-Efficacy in Sedentary Smokers
}

\begin{abstract}
ABSTRACT

Introduction: Cigarette smoking is prevalent with 40 million Americans smoking and one in five deaths attributed to this behavior. Aims: This study examined effects of a resistance training (RT) program on smoking abstinence self-efficacy. Methods: Forty (40) participants were randomized into intervention or control groups. Smoking abstinence self-efficacy, withdrawal symptoms, and smoking behavior were measured. Results/Findings: RT group presented with statistically significant greater increases in selfefficacy compared to control. RT group participants also presented with non-significantly greater decreases in smoking behavior and withdrawal symptoms compared to control. Conclusions: This study demonstrates effectiveness of RT in improving smoking abstinence self-efficacy.
\end{abstract}

\section{Author Bio(s)}

Scott Richardson PT, PhD, is an Assistant Professor in the Doctor of Physical Therapy program in the College of Health and Natural Sciences at Franklin Pierce University.

Brent Alvar PhD, CSCS*D, RSCC*D, FNSCA, FACSM, is a Professor at Point Loma Nazarene University.

Daniel Dodd PhD, is a Professor at Illinois State University.

Uma Nair PhD, is an Assistant Professor at the University of Arizona.

Ryan Seltzer PhD, is Chief Scientific Officer at Translational Analytics and Statistics, Tuscon, Az.

\section{Acknowledgements}

I would like to recognize the immeasurable help and patient support of Dr. Brent Alvar during each stage of the development of this project. 


\title{
IUAHSP \\ The Internet Joutnal of Allied Health Sciences and Practice
}

Dedicated to allied health professional practice and education

Vol. 18 No. 3 ISSN 1540-580X

\section{Effects of Resistance Training on Smoking Abstinence Self-Efficacy in Sedentary Smokers}

\author{
Scott Richardson \\ Brent Alvar \\ Daniel Dodd \\ Uma Nair \\ Ryan Seltzer \\ 1. Franklin Pierce University \\ 2. Point Loma Nazarene University \\ 3. Illinois State University \\ 4. University of Arizona \\ 5. Translational Analytics and Statistics \\ United States
}

\begin{abstract}
Introduction: Cigarette smoking is prevalent with 40 million Americans smoking and one in five deaths attributed to this behavior. Aims: This study examined effects of a resistance training (RT) program on smoking abstinence selfefficacy. Methods: Forty (40) participants were randomized into intervention or control groups. Smoking abstinence self-efficacy, withdrawal symptoms, and smoking behavior were measured. Results/Findings: RT group presented with statistically significant greater increases in self-efficacy compared to control. RT group participants also presented with non-significantly greater decreases in smoking behavior and withdrawal symptoms compared to control. Conclusions: This study demonstrates effectiveness of RT in improving smoking abstinence self-efficacy.
\end{abstract}

Key Words: smoking cessation, self-efficacy, exercise, resistance training 


\section{INTRODUCTION}

Cigarette smoking causes more than 1 in 5 American deaths annually. ${ }^{1}$ This is still a major epidemiological concern, as the Centers for Disease Control reported that in 2014, 17 of every 100 adults (16.8\%) over age 18 in the United States smoke. ${ }^{2}$ Fortunately, $68.8 \%$ of current smokers express a need to quit smoking, and $52.4 \%$ of current adult smokers report an attempt to quit. However, only $6.2 \%$ of smokers report successful cessation in the past year. ${ }^{2}$

When applied to smoking cessation, self-efficacy is described as one's confidence to overcome barriers and successfully quit smoking. ${ }^{3}$ Strong relationships exist between self-efficacy and health behavior change. ${ }^{4}$ Smoking abstinence self-efficacy (SASE) or the confidence to abstain, has a relationship with cessation. ${ }^{5}$ Smokers attempting to quit often consider lifestyle changes to replace harmful behaviors with health- promoting activities such as physical activity/exercise. Participation in leisure time physical activity has been shown to cause improved self-perceived health, while smoking behavior has been shown to decrease selfperception of health. ${ }^{6}$

One of the primary reasons for failure to quit smoking is that smokers experience withdrawal symptoms that can be extremely difficult to overcome. ${ }^{7-8}$ Withdrawal symptoms include irritability/anxiety, insomnia/sleep disorders and tobacco cravings and are a common barrier to cessation.7-10 That stated, exercise has also been shown to assist with withdrawal symptoms and therefore can be useful with cessation effort. ${ }^{11-12}$

Most of the research examining the benefits of exercise with smokers trying to quit has focused on the effects of withdrawal symptoms, sleep patterns, and mood as well as self-esteem. ${ }^{12-13}$ Unfortunately, most of the existing research in this area has only focused on a single mode of physical activity (aerobic exercise) with a majority of the focus on female subjects, as a result of concerns over weight gain with smoking cessation..$^{12}$ Another form of physical activity, resistance training (RT), has promise as an alternate form of physical activity that can assist with smoking cessation. This is because its effects on withdrawal symptoms such as stress reduction and enhanced endorphin release, both of which can decrease irritability and theoretically the urge to smoke. ${ }^{14-}$ 15 In addition, several differences between RT and aerobic exercise may make RT a favorable exercise mode for smokers. Smokers attempting to quit can realize similar exercise benefits utilizing RT versus aerobic exercise while avoiding stresses to the smoker's likely compromised cardiovascular system. ${ }^{15-17}$ Moreover, participation in RT can result in increased muscle mass, increased metabolism, and improved sleep. Preliminary research has shown self-efficacy and/or morale was shown to improve with physical activity in the form of martial arts and with RT. ${ }^{18,19}$ As such, the purpose of this study was to assess the effect of RT on smoking abstinence self-efficacy and withdrawal symptoms.

\section{METHODS \\ Participants}

Participants included male and female smokers, aged $18-50$ (mean 37.52, SD 11.51). Inclusion criteria included a smoking history of 35 or more cigarettes a week for the past 6 months and being sedentary, which was operationalized as scoring "LOW" on the International Physical Activity Questionnaire (IPAQ). The Physical Activity Readiness Questionnaire (PARQ) was also used to gather baseline health information and to screen participants for the study regarding pre-existing health conditions limiting physical activity. ${ }^{20}$ Individuals who reported pre-existing health conditions assessed on the PARQ (e.g. heart disease), were not included in the study.

Additional exclusion criteria included schizophrenia, bipolar disorder or alcohol/drug abuse, pregnancy, or association with the Universities affiliated with the study. Mental health and substance abuse issues were self-reported and excluded due to potential study attrition. Once enrolled, participants randomized to the intervention group provided written physician consent clearing them to participate in strength testing and regular exercise. Study approval was obtained from the Franklin Pierce University Institutional Review Board. Written informed consent was obtained from all participants prior to study participation.

\section{Enrollment}

A total of 162 smokers were referred to the study. Participants entered the study after enrolling in the ASHLine smoking cessation program (32.5\% control, $27.5 \%$ intervention), or by snowballing technique (word of mouth referral) from a study participant (17.5\% control, $22.5 \%$ intervention). Participants were randomized to an exercise or non-exercise control group using a computergenerated list of numbers and assigned to groups during the orientation visit. Participants in either group who enrolled at Arizona Smokers' Helpline (ASHLine) received smoking cessation education, counseling, and nicotine replacement therapy. In addition, individuals enrolling in ASHLine were made aware of a research study and were informed that they could voluntarily contact the study if desired. On the first study visit, participants completed informed consent and baseline testing for efficacy (Smoking Abstinence Self Efficacy-SASE) ${ }^{21}$, and withdrawal symptoms (Mood and Physical Symptoms Scale-MPSS).22 See Figure 1 for a summary of the process from recruitment to data analysis. 


\section{Exercise Group}

Participants in the RT group $(n=20)$ participated in an 8-week RT program on non-consecutive days with each session supervised by the principal investigator for safety and to verify exercise compliance. Supervised sessions were completed at the University Clinic or a local gym. Participants performed a baseline exercise test consisting of a 10-repetition maximum (10RM) for each of the seven study exercises. Each exercise session began with a 5-minute warm-up on a stationary bike without resistance and a simple stretching routine for upper and lower extremities and trunk. RT exercises performed included bench press, bent-over row, overhead triceps extension and biceps curl for the upper extremities, standing squat to parallel, modified dead lift, and calf-raise for the lower extremities.

RT intensity levels were personalized to each individual participant based on his/her current fitness levels. ${ }^{14}$ The American College of Sports Medicine (ACSM) guidelines were followed, with participants completing one set of 10 repetitions at $60 \%$ of their baseline maximal testing during the first 3 weeks. During weeks four to five, participants completed one set of 10 repetitions with weight increased as needed to elicit muscular fatigue. When participants were able to complete 10 repetitions without self-reported substantial fatigue, weight was increased. During weeks six through eight, participants completed two sets of 10 repetitions with weight adjusted as needed to elicit muscle fatigue. Participants rested 90 seconds (measured with a stopwatch) between sets.

Participants were asked not to engage in any RT outside of study exercise sessions to standardize workloads across participants. There was no discussion of smoking, or smoking cessation during RT sessions. RT group participants received self-help materials during visits on week three and six. Self-help materials included information provided by the CDC on deleterious effects of smoking and tips regarding the impact of the social circle of a smoker trying to quit. RT group participants were called weekly after beginning the study and asked for total number of cigarettes smoked each day that week.

\section{Control Group}

Participants in the control group $(n=20)$ completed four total visits. These were orientation (week one) and three additional visits at weeks three, six and eight. Control group participants completed the MPSS and SASE on all visits and received self-help materials (same as RT group) during visits on week three and six. Control group participants were called weekly after beginning the study and asked for total number of cigarettes smoked each day that week.

\section{Measures}

Smoking abstinence self-efficacy (primary outcome), was measured via The Smoking Abstinence Self-Efficacy survey (SASE). This is a valid and reliable tool measuring an individual's confidence to not smoke in various situations (i.e., when angry, when sad, etc.) ${ }^{21}$. Withdrawal symptoms (secondary outcome) were measured via The Mood and Physical Symptom Scale (MPSS) which is a valid and reliable tool measuring irritability, anxiety, restlessness, etc ${ }^{22}$. All assessments were administered to all participants for baseline readings and then every 2 weeks during the 8-week study. Finally, smoking behavior was measured with participants in both groups completing a daily smoking log with this information collected weekly by phone during the study. Quit attempts were operationally defined as report of 0 cigarettes smoked per week following a week when smoking behavior was reported, or a reduction of more than $20 \%$ cigarettes smoked per week.

\section{Data Analysis}

Between group comparisons for age, gender, race, and recruitment method were completed using analysis of variance and Chisquare tests. Post-study results of the outcome measures were assessed using a multivariate analysis of variance to compare group means for cigarettes smoked per week, MPSS, and SASE. Cohen's D effect size analysis was conducted on each of the outcome measures. Post-hoc analysis of smoking abstinence data at study conclusion, number of quit attempts during the study, and withdrawal symptoms after quit-attempts during the study was gathered. Observation of high standard deviations in number of cigarettes smoked between groups led to inclusion of smoking behavior at study entry in the MANOVA.

\section{RESULTS}

A total of 162 smokers were referred to the study. There were 107 potential participants who met inclusion criteria and were not excluded per study criteria. Of these, 61 obtained physician clearances to participate, with 36 randomized to the exercise group and 22 to the control group. Between group comparisons for age, gender, race, and recruitment were completed with no significant differences between groups (Table 1). Participants were predominately female (77.5\%) with a mean age of 37.5 years (SD 11.51). Participants were $75 \%$ Caucasian, $12.5 \%$ African-American, $10 \%$ Hispanic and $2.5 \%$ Pacific Islander. Sources of referral to the study were $60 \%$ from ASHLine, and $40 \%$ via word-of-mouth. There were 36 total participants randomized to the exercise group with reasons for 16 subjects leaving study because of driving distance to exercise sessions and the lack of available time off work. There were 22 total participants randomized to the control group with 2 leaving the study because of work schedule conflicts. Final 
participants in both exercise and control groups were 20 in each group.

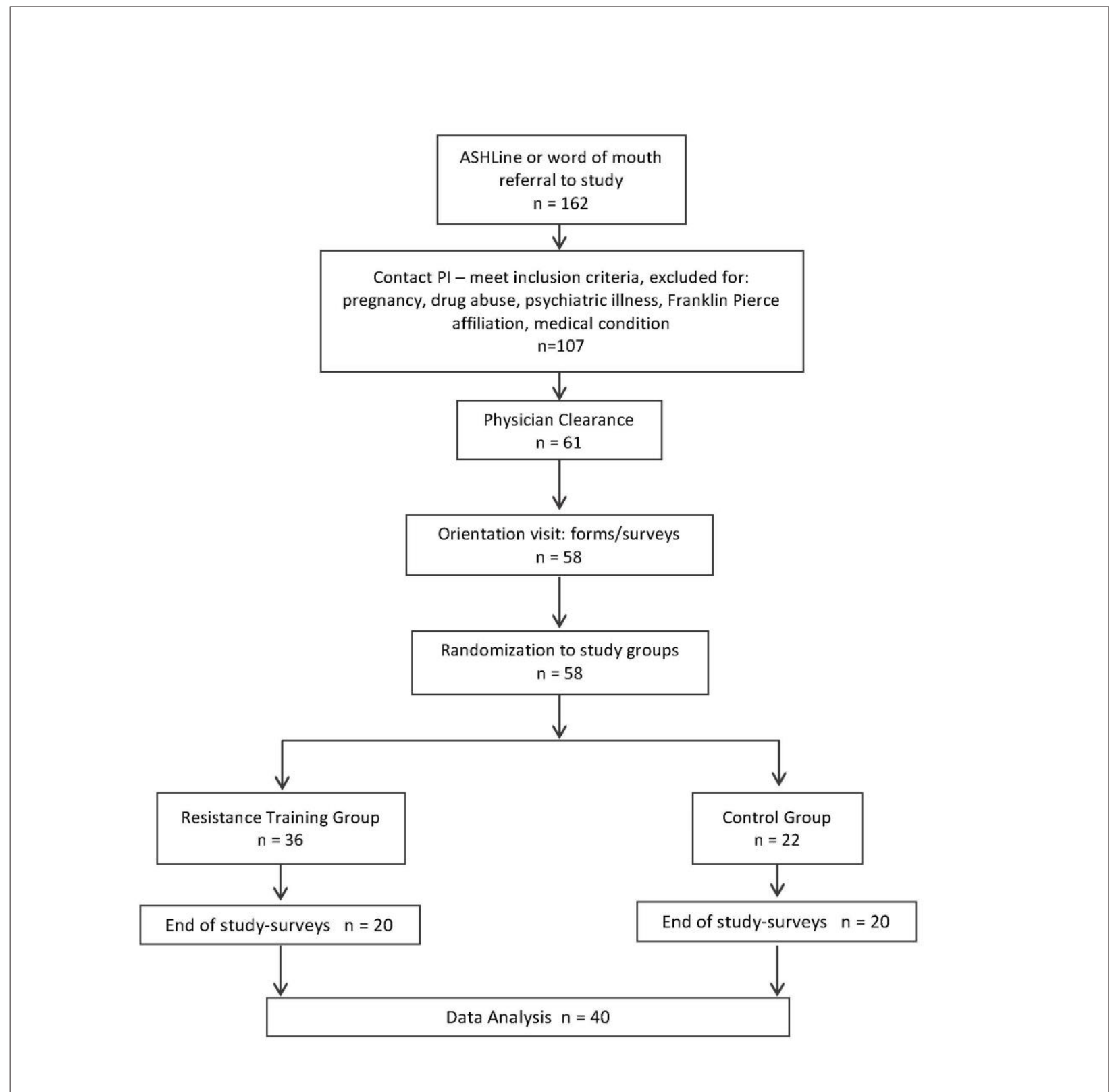

Figure 1. Study Flow Sheet

\section{RESULTS}

A total of 162 smokers were referred to the study. There were 107 potential participants who met inclusion criteria and were not excluded per study criteria. Of these, 61 obtained physician clearances to participate, with 36 randomized to the exercise group and 22 to the control group. Between group comparisons for age, gender, race, and recruitment were completed with no significant differences between groups (Table 1). Participants were predominately female (77.5\%) with a mean age of 37.5 years (SD 11.51). Participants were $75 \%$ Caucasian, $12.5 \%$ African-American, 10\% Hispanic and $2.5 \%$ Pacific Islander. Sources of referral to the study were $60 \%$ from ASHLine, and $40 \%$ via word-of-mouth. There were 36 total participants randomized to the exercise group with reasons for 16 subjects leaving study because of driving distance to exercise sessions and the lack of available time off work. There were 22 total participants randomized to the control group with 2 leaving the study because of work schedule conflicts. Final participants in both exercise and control groups were 20 in each group. 
Table 1: Participant Demographics

\begin{tabular}{c|ccc}
\hline & CONTROL & INTERVENTION & P VALUE \\
\hline GENDER & Females 13 & Females 18 \\
& Males 7 & Males 2 & .06 \\
AGE & & & .97 \\
(MEAN) & $37.45+/-12.28$ & $37.60+/-10.99$ & \\
& Caucasian 13 & & .43 \\
RACE & Black 3 & Caucasian 17 \\
& Hispanic 3 & Black 2 & \\
STUDY & Hispanic 1 & .52 \\
ENTRY & Referral 7 & 11 & \\
\hline
\end{tabular}

Assessment of outcome measures was conducted with a multivariate analysis of variance to compare group means to measure between group differences in smoking abstinence self-efficacy (SASE), withdrawal symptoms (MPSS), and number of cigarettes smoked (Table 2). Significance was achieved with assessment of smoking abstinence self-efficacy, with intervention group participants demonstrating greater self-efficacy compared to control group participants at end of study ( $p<.0001)$. Smoking behavior decreased in both groups, however, there were no significant differences between groups $(p=.23)$. In addition, withdrawal symptoms scores (MPSS) also decreased in both groups with no significant differences between groups ( $p=.45)$. Cohen's $D$ effect size analysis was conducted on each of the outcome measures. The effect sizes were 2.24 (large) for smoking abstinence selfefficacy (SASE) favoring the resistance training group, .14 (small) for cigarettes smoked, and .03 (small) for withdrawal symptoms (MPSS). In addition, the exercise group participants made 32 quit or reduction attempts during the 8-week study (report of 0 cigarettes smoked per week following a week when smoking behavior was reported, or a reduction of more than $20 \%$ cigarettes smoked per week), compared to 18 quit-attempts by control group participants ( $p=.65)$.

To account for the possibility of changes between groups with smoking behavior that may have occurred during the 8-week study, but perhaps were not maintained at study conclusion, a week-by-week comparison was done with no significant differences found. This was done with a series of t-tests comparing differences between groups for cigarettes smoked during consecutive weeks with p values ranging from .051 to .95 with Bonferroni correction applied for the 7 analyses.

Table 2. Summary of Results

\begin{tabular}{c|ccccc}
\hline \multicolumn{1}{c}{} & CONTROL & INTERVENTION & F & P VALUE & $\begin{array}{c}\text { EFFECT } \\
\text { SIZE }\end{array}$ \\
\hline $\begin{array}{c}\text { TOTAL } \\
\text { CIGARETTES }\end{array}$ & $-5.95+/-20.93$ & $-8.95+/-12.60$ & 1.453 & .23 & .14 \\
MPSS & $-2.45+/-8.19$ & $-2.70+/-6.97$ & .977 & .45 & .03 \\
& & & & & \\
SASE & $1.10+/-2.45$ & $-4.40+/-2.06$ & 11.550 & $<.0001$ & 2.24 \\
\hline
\end{tabular}

\section{DISCUSSION/CONCLUSIONS}

Smoking continues to be the leading preventable cause of death and disease. Smokers trying to quit face many challenges, including managing social interactions that may affect urges to smoke, life stressors, and resulting coping mechanisms. For these 
reasons, measurement and consideration of smoking abstinence self-efficacy is crucial to understanding the perceived barriers for a given smoker and the triggers that can cause relapse. Results indicate significantly higher efficacy in RT participants. Our results show that RT can help increase smoking abstinence self-efficacy.

Most exercise related smoking cessation studies focus on smoking abstinence. The present study examined effects of RT on smoking abstinence self-efficacy, or the confidence related to cessation. Resistance-training group participants reduced overall number of cigarettes smoked per week, were involved in an ongoing exercise program, and reported significantly improved smoking abstinence self-efficacy. This has importance because these participants report significantly improved confidence in their abilities to remain abstinent in various situations that would typically cause relapse. ${ }^{21}$ Active participation in an RT program appears to serve as a healthy replacement behavior for smoking and perhaps explains the improved smoking abstinence self-efficacy. In addition, even though the differences between groups did not achieve statistical significance, another indicator of successful smoking cessation intervention was the greater number of quit attempts in the resistance training group. ${ }^{23}$

\section{Limitations}

There are a few limitations to the present study which need to be discussed. To ensure efficacy of the intervention, baseline testing and structured progression of frequency and volume of exercises was utilized. One explanation for the lack of statistical significance with measurement of withdrawal symptoms is the transitory nature of the intervention effect. Studies have shown that exercise can reduce withdrawal symptoms; however, the benefits are fairly acute and have been reported to not persist for an extended duration after an exercise bout.24,25 Participants in the present study only exercised twice weekly, with little to no control over the amount of time between exercise sessions and the reported withdrawal symptoms. This was a definite drawback in the study design, causing potential failure to capture timing of the intervention effects on withdrawal symptoms.

\section{Recommendations for Future Research}

Future research might consider utilizing RT with a more flexible, lifestyle physical activity approach, encouraging more frequent bouts of exercise generally as well as when withdrawal symptoms are experienced.24-27 Research has shown an increased adherence to exercise programs with multiple short bouts of exercise. ${ }^{28}$ Perhaps more frequent involvement with RT would yield greater reductions in withdrawal symptoms and decreased smoking behavior.

Additional considerations for future research include a mixed-methods approach to interview subjects and the exploration of attitudes of smokers engaged in RT. This would increase understanding of the subjects' true experience while utilizing RT as a component of the intervention. In addition, collection of behavior change data could also provide insights into factors affecting the increased smoking abstinence self-efficacy in RT participants in the present study. Finding subjects who were at the Action Stage of Prochaska's Transtheoretical Model would clarify whether the subjects were indeed ready for such an intervention. The results of this study provide insight into the benefits of RT as an option for individuals desiring to quit smoking.

\section{References}

1. Siegel R, Ma J, Zou Z, Jemal A. Cancer statistics, 2014. CA Cancer J Clin. 2014; 64(1): 9-29. DOI: 10.3322/caac.21208

2. CDC. Quitting smoking among adults-United States, 2001-2010. Morbidity and Mortality Weekly Report. 2011; 60(44).

3. Prochaska JO, Velicer WF. The transtheoretical model of health behavior change. AM J Health Promot. 1997; 12(1): 38-48. DOI: 10.4278/0890-1171-12.1.38

4. Stretcher VJ, DeVellis BM, Becker MH, Rosenstock IM. Self-efficacy and the health belief model. Health Educ Q. 1986; 13: 73-92. DOI: 10.1177/109019818601300108

5. Gwaltney CJ, Metrik, J, Kahler CW, Shiffman S. Self-efficacy and smoking cessation: a meta-analysis. Psychol Addict Behav. 2009; 23(1): 56. DOI: 10.1037/a0013529

6. Okano G, Miyake H, Mori M. Leisure time physical activity as a determinant of self-perceived health and fitness in middleaged male employees. J Occup Health. 2003; 45(5): 286-292. DOI: 10.1539/joh.45.286

7. Aguilar AJ, DiStefano LJ, Brown CN, Herman DC, Guskiewicz KM, Padua DA. A dynamic warm-up model increases quadriceps strength and hamstring flexibility. J Strength Cond Res. 2012;26: 1130-1141. DOI:10.1519/JSC.0b013e31822e58b6.

8. Albrecht SA, Caruthers D, Patrick T, Reynolds M, Salamie D, Higgins LW, Braskte B, Kim Y, Mlynarchek S. A randomized controlled trial of a smoking cessation intervention for pregnant adolescents. Nurs Res. 2006;55: 402-410. DOI: 10.1097/00006199-200611000-00004

9. Alamar B, Glantz SA. Effect of increased social unacceptability of cigarette smoking on reduction in cigarette consumption. Am J Public Health. 2006; 96(8): 1359-1363. DOI: 10.2105/AJPH.2005.069617 
10. Asher MK, Martin RA, Rohsenow DJ, MacKinnon SV, Traficante R, Monti PM. Perceived barriers to quitting smoking among alcohol dependent patients in treatment. J Subst Abuse Treat. 2003; 24(2): 169-174. DOI: 10.1016/s0740-5472(02)00354-9

11. Byron-Daniel JZ, Cropley M, Fife-Schaw $C$. The effect of exercise in reducing desire to smoke and cigarette withdrawal symptoms is not caused by distraction. Addiction. 2006; 101: 1187-1192. DOI:10.1111/j.1360-0443.2006.01457.x

12. Taylor $\mathrm{AH}$, Ussher $\mathrm{MH}$, Faulkner $\mathrm{G}$. The acute effects of exercise on cigarette cravings, withdrawal symptoms, affect and smoking behaviour: a systematic review. Addiction. 2007; 102: 534-543. DOI:10.1111/j.1360-0443.2006.01739-x.

13. DeRuiter WK, Faulkner G, Cairney J, Veldhuizen, S. Characteristics of physically active smokers and implications for harm reduction. Am J Public Health. 2008; 98(5): 925-931. DOI: 10.2105/AJPH.2007.120469

14. Kraemer WJ, Adams K, Cafarelli E, et al. American College of Sports Medicine position stand. Progression models in resistance training for healthy adults. Medicine and Science in Sports and Exercise. 2002; 34(2): 364-380. DOI: 10.1097/00005768-200202000-00027

15. Ciccolo JT, Dunsiger SI, Williams DM, et al. Resistance training as an aid to standard smoking cessation treatment: A pilot study. Nicotine Tob Res. 2011; 13(8): 756-760. DOI: 10.1093/ntr/ntr068

16. Asthana $A$, Piper ME, McBride $P E$, et al. Long-term effects of smoking and smoking cessation on exercise stress testing: three-year outcomes from a randomized clinical trial. AM Heart J. 2012; 163(1): 81. DOI:10.1016/j.ahj.2011.06.023.

17. Crow RS, Rautaharju PM., Prineas RJ et al. Risk factors, exercise fitness and electrocardiographic response to exercise in 12,866 men at high risk of symptomatic coronary heart disease. Am J Cardiol. 1986; 57(13): 1075-1082. DOl:https://doi.org/10.1016/0002-9149(86)90677-6

18. Bodi T, Martinsen EW. Mood and self-efficacy during acute exercise in clinical depression: A randomized, controlled study. J Sport Exercise PSY. 2004; 26(4): 623-633. DOI: 10.1123/jsep.26.4.623

19. Singh NA, Clements KM, Fiatarone MA. A randomized controlled trial of progressive resistance training in depressed elders. J Gerontol A Biol Sci Med Sci.1997; 52(1): M27-M35. DOI: 10.1093/gerona/52a.1.m27

20. Warburton DE, Jamnik,VK, Bredin SS, Gledhill, N. The physical activity readiness questionnaire for everyone (PAR-Q+) and electronic physical activity readiness medical examination (ePARmed- $X+$ ). The Health \& Fitness Journal of Canada (HFJC). 2011; 4(2): 3-17. DOI: https://doi.org/10.14288/hfjc.v4i2.103

21. Spek V, Lemmens F, Chatrou M, vanKempen S, Pouwer F, Pop V. Development of a smoking abstinence self-efficacy questionnaire. Int J Behav Med. 2013; 20(3): 444-449. DOI: 10.1007/s12529-012-9229-2

22. West R, Hajek P. Evaluation of the mood and physical symptoms scale (MPSS) to assess cigarette withdrawal. Psychopharmacology (Berlin). 2004; 177(1-2): 195-199. DOI:10.1007/s00213-004-1923-6.

23. Hymowitz N, Cummings KM, Hyland A, Lynn WR, Pechacek TF, Hartwell TD. Predictors of smoking cessation in a cohort of adult smokers followed for five years. BMJ Group. 1997. DOI: 10.1136/tc.6.suppl_2.s57

24. Scerbo F, Faulkner G, Taylor A, Thomas S. Effects of exercise on cravings to smoke: the role of exercise intensity and cortisol. J SPORT SCI MED. 2009; 28: 11-19. doi:10.1080/02640410903390089.

25. Ussher M, Nunziata $P$, Crople, $M$, West $R$. Effect of a short bout of exercise on tobacco withdrawal symptoms and desire to smoke. Psychopharmacology (Berlin). 2001; 158: 66-72. DOI:10.1007/s002130100846.

26. Daniel J, Cropley M, Ussher M, Wes, R. Acute effects of a short bout of moderate versus light intensity exercise versus inactivity on tobacco withdrawal symptoms in sedentary smokers. Psychopharmacology. 2004; 174: 320-326. DOI:10.1007/s00213-003-1762-X.

27. Linke SE, Rutledge T, Myers MG. Intermittent exercise in response to cigarette cravings in the context of an Internet-based smoking cessation program. Ment Health Phys Act. 2012; 5(1): 85-92. DOI: 10.1016/j.mhpa.2012.02.001

28. Jakicic JM, Wing RR, Butler BA, Robertson RJ. Prescribing exercise in multiple short bouts versus one continuous bout: effects on adherence, cardiorespiratory fitness, and weight loss in overweight women. Int J Obes Relat Metab Disord. 1995; 19: 893-901. PMID: 8963358 